\title{
Gaps in Study Design for Immune Parameter Research for Latent Tuberculosis Infection: A Systematic Review
}

\author{
Mariana Herrera $\mathbb{D}^{\mathrm{D}},{ }^{1,2}$ Cristian Vera $\mathbb{D}^{\mathrm{D}},{ }^{2,3}$ Yoav Keynan $\mathbb{D}^{4},{ }^{4}$ and Zulma Vanessa Rueda ${ }^{2,5}$ \\ ${ }^{1}$ Grupo de Epidemiología, Facultad Nacional de Salud Pública, Universidad de Antioquia, Medellín, Colombia \\ ${ }^{2}$ Grupo de Investigación en Salud Pública, Universidad Pontificia Bolivariana, Medellín, Colombia \\ ${ }^{3}$ Clínica Universitaria Bolivariana, Universidad Pontificia Bolivariana, Medellín, Colombia \\ ${ }^{4}$ Departments of Internal Medicine, Medical Microbiology \& Infectious Diseases and Community Health Sciences, \\ University of Manitoba, Winnipeg, Canada \\ ${ }^{5}$ Facultad de Medicina, Universidad Pontificia Bolivariana, Medellín, Colombia
}

Correspondence should be addressed to Zulma Vanessa Rueda; zulmaruedav@gmail.com

Received 10 November 2019; Accepted 16 March 2020; Published 21 April 2020

Academic Editor: Eirini Rigopoulou

Copyright (c) 2020 Mariana Herrera et al. This is an open access article distributed under the Creative Commons Attribution License, which permits unrestricted use, distribution, and reproduction in any medium, provided the original work is properly cited.

\begin{abstract}
Background. Immune parameters (IP) have been extensively studied to distinguish between latent tuberculosis (LTBI) and active tuberculosis (TB). Objective. To determine the IP associated with LTBI, compared to active TB and individuals not infected by M. tuberculosis published in literature. Methods. We conducted a systematic search using Google Scholar and PubMed databases, combining the MeSH terms latent tuberculosis, Mycobacterium tuberculosis, cytokines, and biological markers, with the free terms, biomarkers and cytokines. Spanish, English, and Portuguese articles comparing the concentration of IP associated with LTBI, either in plasma/serum or in vitro, in adults and nonimmunocompromised versus individuals with TB or without M. tuberculosis infection between 2006 July and 2018 July were included. Two blinded reviewers carried out the searches, read the abstracts, and selected the articles for analysis. Participants' information, diagnostic criteria, IP, detection methods, and biases were collected. Results. We analyzed 36 articles (of 637 abstracts) with 93 different biomarkers in different samples. We found 24 parameters that were increased only in active TB (TGF- $\alpha$, CSF3, CSF2, CCL1 [I-309], IL-7, TGF- $\beta 1$, CCL3 [MIP-1 $\alpha$ ], sIL-2R, TNF- $\beta$, CCL7 [MCP-3], IFN- $\alpha$, fractalkine, I-TAG, CCL8 [MCP-2], CCL21 [6Ckine], PDGF, IL-22, VEGF-A, LXA4, PGE2, PGF2 $\alpha$, sCD163, sCD14, and 15-Epi-LXA4), five were elevated in LTBI (IL-5, IL-17F, IL-1, CCL20 $[\mathrm{MIP}-3 \alpha]$, and ICAM-1), and two substances were increased among uninfected individuals (IL-23 and basic FGF). We found high heterogeneity between studies including failure to account for the time/illness of the individuals studied; varied samples and protocols; different clinical classification of TB; different laboratory methods for IP detection, which in turn leads to variable units of measurement and assay sensitivities; and selection bias regarding TST and booster effect. None of the studies adjusted the analysis for the effect of ethnicity. Conclusions. It is mandatory to harmonize the study of immune parameters for LTBI diagnosis. This systematic review is registered with PROSPERO CRD42017073289.
\end{abstract}

\section{Background}

Latent tuberculosis (LTBI) is defined as the presence of a positive tuberculin skin test (TST) or interferon- $\gamma($ IFN $\gamma$ ) release assays (IGRAs) in the absence of clinical or radiographic signs of disease. These accepted tests are imperfect for LTBI diagnosis for several reasons: (1) the sensitivity and specificity are between 71 and $82 \%$ for TST and 81 and $86 \%$ for
IGRAs [1], (2) the sensitivity is reduced in immunocompromised patients, (3) there is inability to differentiate between LTBI and active tuberculosis (TB), (4) a positive TST or IGRA result does not automatically imply LTBI, as individuals who eliminate the infection successfully might still be TST- or IGRA-positive because of memory T cell responses, which partly explains the low predictive value of TST and IGRAs [1], and (5) genetic factors may impact test sensitivity 
as well as the susceptibility for acquisition of mycobacterial infection [2-4]. To date, there is no available diagnostic tool that allows diagnosis of LTBI and differentiates clearly between LTBI and active TB.

For the above-mentioned reasons, the World Health Organization, governments and nongovernmental organization, and private sector established as one of the priorities the identification of "what biomarkers or combinations of markers will help distinguish the various stages of the spectrum of LTBI (from sterilizing immunity to subclinical active disease)" [5].

The improvement in high-throughput cytokine measurement platforms has sparked enthusiasm for identification of novel pathways involved in the pathogenesis of TB that can inform development of assays for LTBI determination. In tuberculous infection, some important immune molecules are known to play a pivotal role in the protective response against the bacteria. Among the main ones described are IFN- $\gamma$, produced by T CD4+, CD $8+$, and NK cells, and IL- 1 and TNF- $\alpha$, secreted by macrophages and lymphocytes, known to prevent the growth and multiplication of mycobacteria in host cells $[6,7]$. However, additional biomarkers such as IL-2, IL-5, IL-10, IL-1RA, and MCP have been studied for their ability to differentiate between the LTBI and active TB [8], and it is believed that the cellular and immune profile expressed during tuberculous infection depends to a great extent on the stage of disease, i.e., LTBI or active, where immune biomarkers present in blood could have the ability to differentiate with greater precision between both stages [9].

Despite advances in the study of immune parameters, there are pervasive limitations in the analysis and conclusions of many of these studies. Cytokine/chemokine expression is affected by ethnicity $[2,10]$, cell simulation protocols (or no stimulation) [11-13], time of LTBI (which in most cases is impossible to quantify), and if the comparison group is people with TB, the clinical manifestations of disease (pulmonary vs. extrapulmonary TB) [14].

In order to identify which immune parameters are increased exclusively in LTBI, in addition to finding gaps in knowledge and study design of previous published papers, we performed a systematic review. The question posed is the following: what are the cytokines associated with LTBI, compared to cytokines expressed among individuals with active TB and those not infected by $M$. tuberculosis?

\section{Methods}

According to the Preferred Reporting Items for Systematic reviews and Meta-Analysis protocols (PRISMA-P), this systematic review was registered with the International Prospective Register of Systematic Reviews (PROSPERO) on August 31, 2017 (registration number CRD42017073289).

2.1. Eligibility Criteria. Studies were selected according to the following criteria.

2.1.1. Study Designs. We included clinical trials, prospective and retrospective comparative cohorts, and case-control and cross-sectional studies. We excluded descriptive studies, case reports and series, and reviews.

2.1.2. Participants. The participants are those from articles published between January 2006 and July 2018, which compared people with LTBI with 18 years or older, or adults and children, without any immunocompromising medical conditions, versus individuals with active TB or without $M$. tuberculosis infection under the same conditions. We excluded manuscripts assessing the production of IFN- $\gamma$ as part of the evaluation of IGRAs, which were performed in animal models, immunocompromised individuals, and studies exclusively conducted in children.

2.1.3. Exposure. Articles that evaluated the expression of cytokines associated with LTBI, either in plasma or in vitro, with or without stimulation of mycobacterial antigens were included. The antigens used to perform cell stimulation were not restricted.

2.1.4. Comparators. The comparators are the expression of cytokines associated with active TB confirmed by clinical and epidemiological contact, X-rays, and/or laboratory and/or subjects with no evidence of $M$. tuberculosis infection, evidenced by negative results of the tuberculin skin test or interferon-gamma release assays.

2.1.5. Outcome. People with LTBI were compared to those with active TB or with no evidence of $M$. tuberculosis.

2.1.6. Timing. There was no restriction on the length of follow-up for clinical trials or cohort studies.

\subsubsection{Setting. There was no restriction on the type of setting.}

2.1.8. Language. Articles in English, Spanish, or Portuguese were included.

2.2. Information Sources. Search for original articles utilized two electronic databases: Google Scholar and PubMed.

To identify additional literature, the reference list of all papers was reviewed, and we followed the same process for abstract reviewing and data extraction as we did for papers identified by electronic search. Articles suggested by the reviewers, not detected in the previous searches, were also included.

2.3. Search Strategy. Papers published between July 2006 and July 2018 were included. We used the following MeSH terms in English, Spanish, and Portuguese languages: latent tuberculosis, Mycobacterium tuberculosis, cytokines, and biological markers. In addition, we used the free terms biomarkers and cytokines. Additional file 1 contains the search strategies used.

2.4. Study Selection, Data Collection Process, and Data Items. Once the articles were identified using each of the search strategies, we proceeded with the elimination of duplicate items. Subsequently, the titles and abstracts of all manuscripts identified by two independent evaluators were reviewed according to the selection criteria. All disagreements between 
the two reviewers were resolved with a third evaluator by consensus. Articles that met the selection criteria were read completely by the same reviewers, blinded and independently.

The data extracted and typed in an Excel file from the selected articles were the following: consecutive number of the article (whole number assigned by investigators), article title, year, first author, journal, study country of origin, outcome or result reported in the article, type of study population (special feature), number of patients in the intervention or comparison group, follow-up in each group, type of control or unexposed population, number of patients in the control or unexposed group, follow-up in the control or nonexposed group (months), age, sex (female percentage), active TB diagnostic method, LTBI diagnostic method, LTBI time, immune parameters studied, increased IP (with and without statistical differences) (the group in which the IP was increased is reported first), IP that remained normal, decreased IP (with or without statistical differences), IP concentration values, the level of confidence they used in their statistical analyses (90\%, 95\%, and 99\%), method of detection of IP, if ethnicity was reported, the study populations, type of study, quality of the study (see below), bias (types of bias), proportion of BCG vaccine, conflict of interest statement, and other important findings such as the cell stimulation used (times and antigens used).

We conducted a pilot study for the search strategies, abstract reviewing, and data extraction of full-text articles to standardize all process and concepts before starting each step. A third reviewer was in charge of comparing the files to identify disagreements at each step of the process. A fourth reviewer participated in the validation of the biological findings, only at the end of the full-data extraction for included papers to avoid investigator bias.

2.5. Risk of Bias in Individual Studies. Selection bias was controlled through the application of inclusion and exclusion criteria to eligible titles and/or summaries; likewise, possible information biases were controlled by the independent revision of two observers, where at the end of the review, a third reviewer compared their findings. The risk of bias of the studies was assessed using the Newcastle-Ottawa scales for casecontrol and cohort studies [15] and the National Institutes of Health $(\mathrm{NIH})$ evaluation scale for observational studies [16]. The Jadad scale was applied to evaluation of clinical trials [17] (Additional files 3 and 4).

The Newcastle-Ottawa scale evaluates four main points: population, that is, the choice of cases or exposed people, and controls or not exposed; the measurement of the outcome and exposure; and the comparability between groups [15]. Similarly, the NIH scale is based on 14 questions that include the clear definition of the objective, the population (including the sample size), the measurement of dependent and independent variables, and the control of the confounders [16].

For both scales, one or two points are given when a study complies with the evaluated requirements (comparability for Newcastle-Ottawa). This final score determines the risk of bias: high risk (0-2 points), moderate (between 3 and 6 points), and low risk of bias ( $\geq 7$ points).
2.6. Summary Measures. Due to the clinical heterogeneity of the population, the samples and the stimulation protocol used, the multiple techniques used for immune parameter detection, the different units reported for the substances, and the differences in the diagnosis of LTBI and active TB, it is was deemed inadequate to perform a meta-analysis [18, 19]. Therefore, we report the systematic review with a qualitative synthesis of the papers.

\section{Results}

3.1. Articles. Upon searching according to the keywords, 637 relevant articles were retrieved; among them, 58 met the selection criteria and were read in full text. At the end, 36 met all criteria and were included in the systematic review (Figure 1). The excluded articles and the reasons for exclusion are provided in Additional file 2.

Publications included 34 cross-sectional and 2 cohort studies, the latter with follow-up for 6 and 24 months after baseline sampling.

3.2. Participants. Most of the studies evaluated individuals with active TB treatment or within hospital programs. Their community or family contacts or voluntary hospital or community-based controls with or without TB infection served as controls. Five studies were conducted in healthcare workers, four in places endemic for $\mathrm{TB}$, and one from a region with a high rate of malnutrition. The minimum and maximum numbers of subjects included in the studies were 7 and 148 in the LTBI group, 10 and 147 in the active TB group, and 8 and 168 in the noninfected group. Table 1 describes the characteristics of the population included in each study.

3.3. BCG Vaccination Status. Among the 36 included articles, 22 reported BCG vaccination [20-42]. The proportion of BCG vaccination was similar among the groups with LTBI, active TB, and noninfected individuals. Seven papers [26, $28,36,38,42-44]$ compared the statistical difference between those with and without vaccination, and only two reported statistical significance $[45,20]$, having a lower percentage in the active TB group compared to healthy controls (healthy persons with no known risk of TB exposure), TB-exposed persons with QFT-negative results, and people with LTBI. One article reported no significant associations between levels of cytokines and BCG scar [21], and one included BCG status in the multinomial regression model getting an adjusted odds ratio increased for TNF- $\alpha$ and IL-6 [22].

3.4. Evaluation of Conversion to LTBI and Progression to Active TB. The majority of the studies did not evaluate progression to active TB and conversion to LTBI. Most of literatures that we reviewed were cross-sectional studies that only have the prevalence or frequency of LTBI $[22,21]$ and active TB and those who are TST-negative or IGRA-negative. We found two cohort studies, and only one of them evaluated risk of LTBI conversion and progression to diseases, but it reported that they have a low number of convertor $(2 / 101$ individuals) and any progressor to active TB [21]; therefore, 


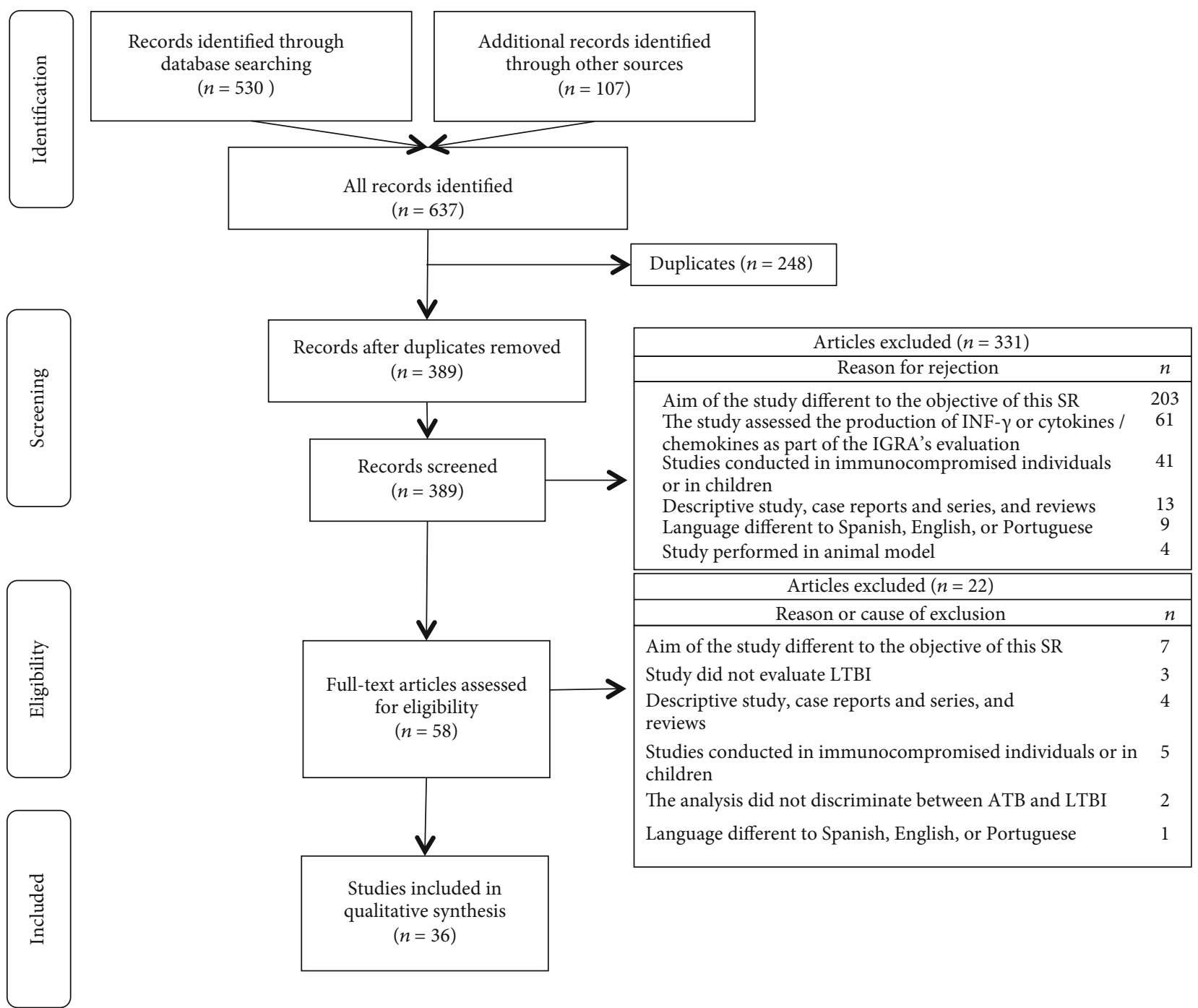

FIGURE 1: PRISMA diagram showing the results of systematic searches and articles analyzed. Legend: ATB: active tuberculosis; LTBI: latent tuberculosis infection.

it is not feasible to identify cytokines that allow to identify progression to either.

3.5. Diagnostic Methods for LTBI and Active TB. The methods used for LTBI diagnosis were the following: 18 studies used TST and IGRAs, nine relied on TST alone, one used TST or IGRAs plus clinical criteria, and eight utilized IGRAs alone. In studies where the two tests were used, the discordant results between the two tests are evident.

In order to establish the diagnosis of active $\mathrm{TB}$, researchers used one or a combination of the following criteria: history of contact with a TB case, smear (Ziehl-Neelsen or auramine rhodamine stain), culture, clinical diagnosis, molecular test, pathology, and/or X-rays.

3.6. Measurement of Immune Parameters. In total, 93 substances (Table 2) were studied, including growth factors; interferons; receptors; tumor necrosis factors; alpha, beta, and delta chemokines; interleukins; and others like sCD40L, MIF, and sCD14.
Of these, 24 substances were increased only in active TB, five increased only in the LTBI group, and two increased in uninfected individuals, regardless of the sample analyzed (Figure 2).

Table 1 shows all the antigens and times used for ex vivo stimulation and cytokines whose concentration was statistically different, by each group. The most frequently measured mediators were IL-6, IL-10, IL-2, TNF- $\alpha$, INF- $\gamma$, and IP-10.

Most of the studies reported mediators after ex vivo stimulation, with most using supernatants of interferon-gamma release assays.

Only eight papers evaluated serum or plasma samples without stimulation. There were no differences between them; only MIG was increased in plasma samples in TB patients, but elevated in serum samples in both, LTBI and active TB.

Among evaluated immune mediators, most were measured in plasma samples from stimulated or unstimulated (as controls) whole blood (Figures 2(a)-2(g)). Other samples utilized were supernatants from PBMC cultures and whole blood culture and RNA from blood cells. 


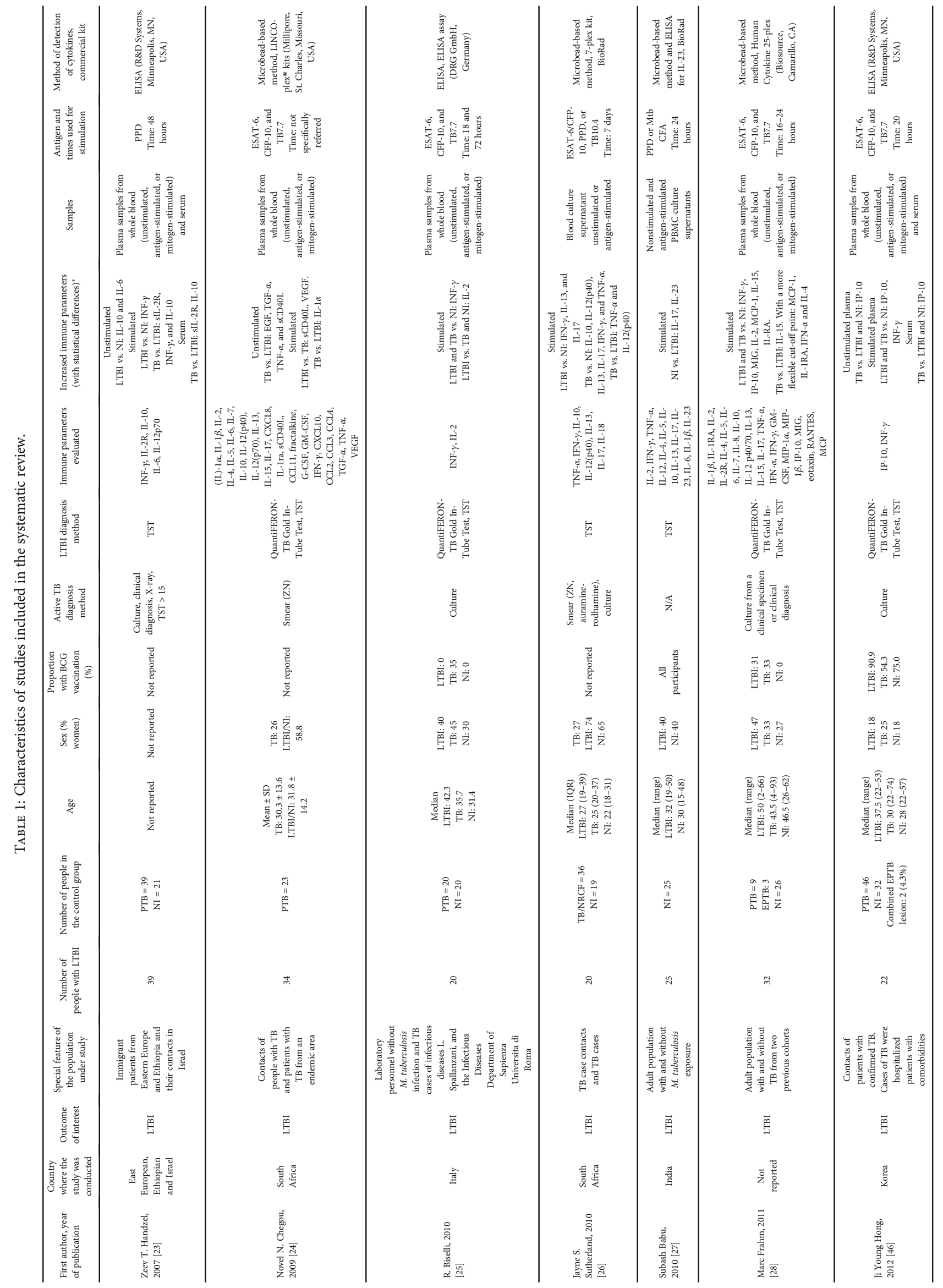




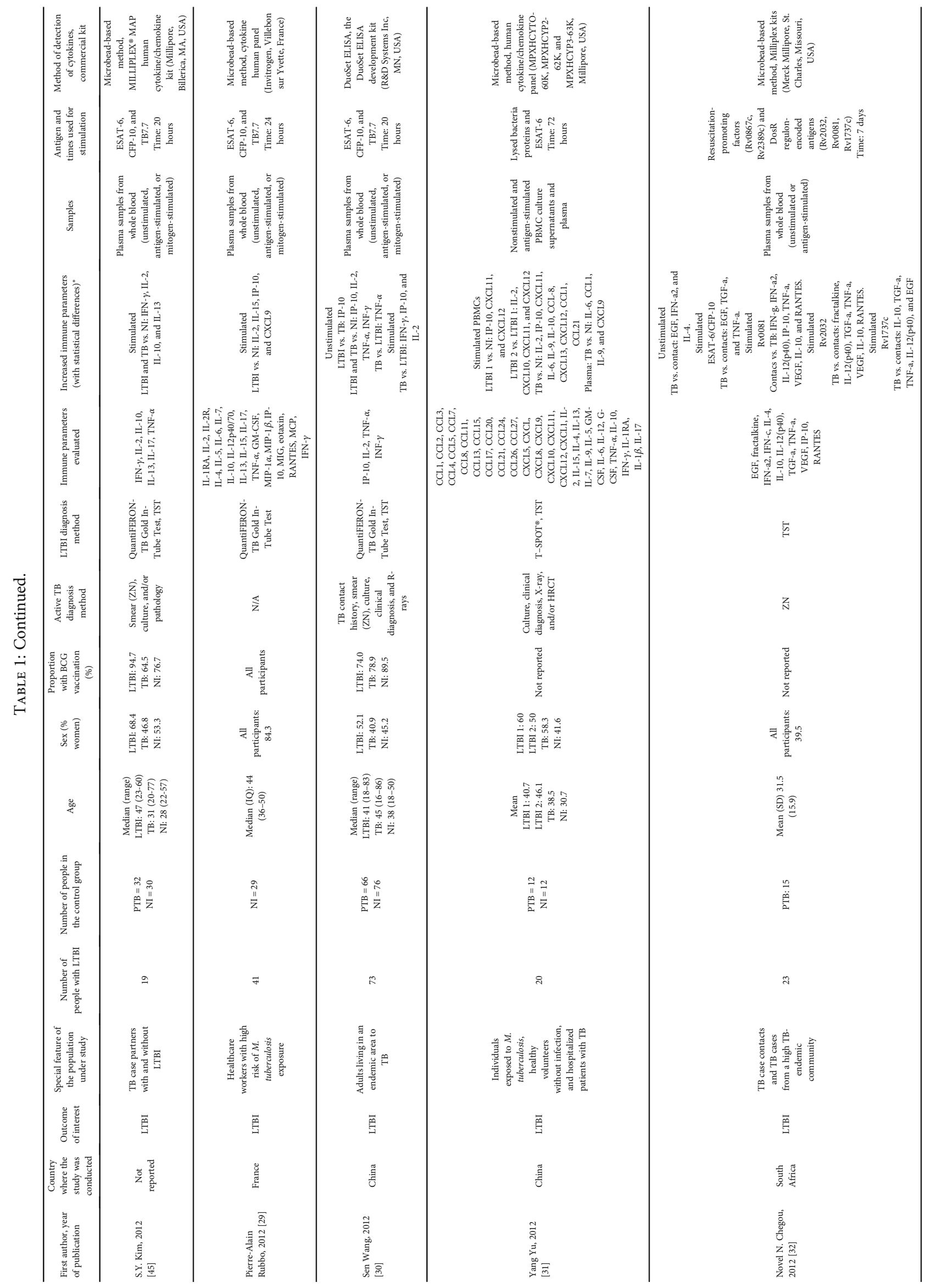




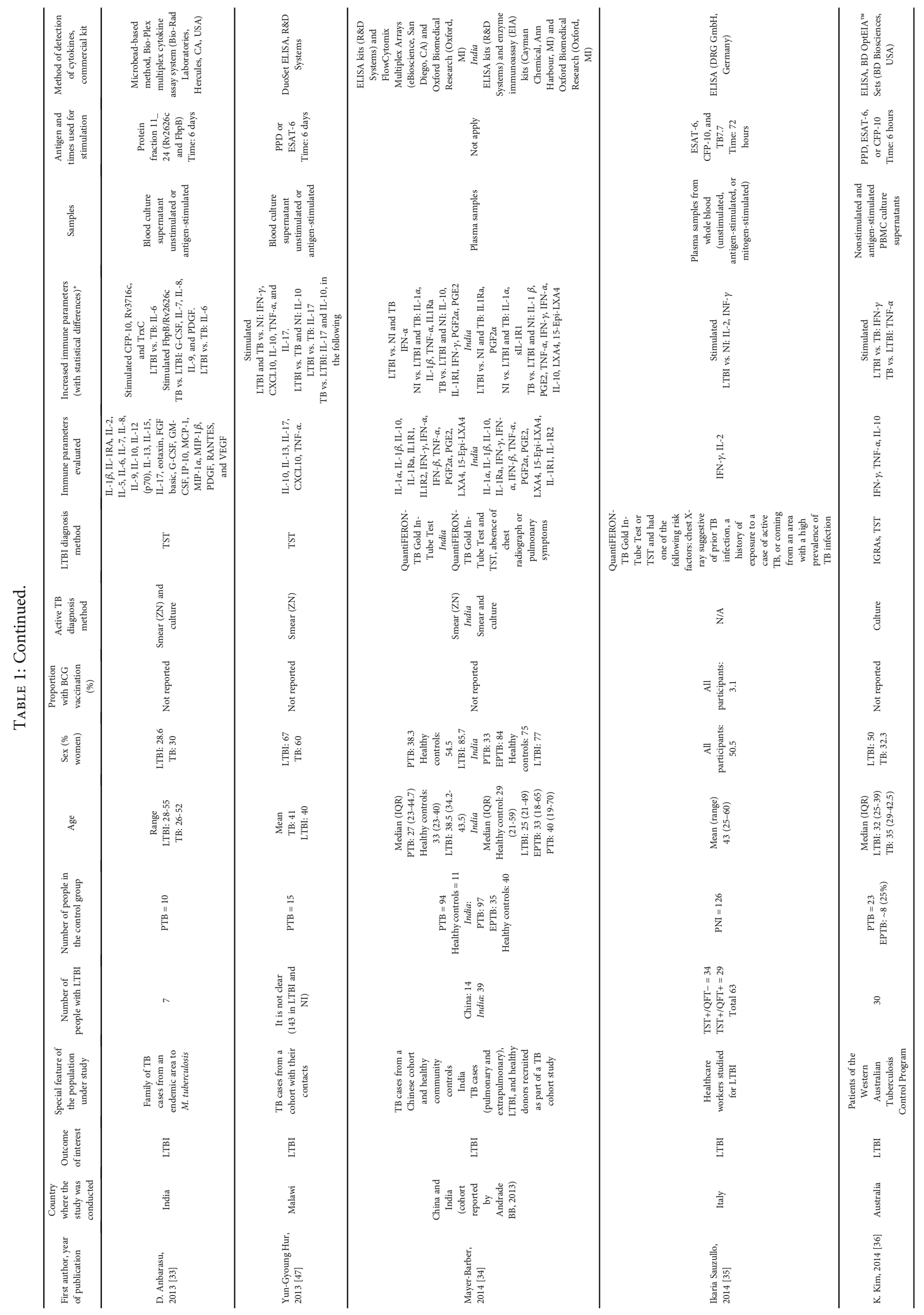









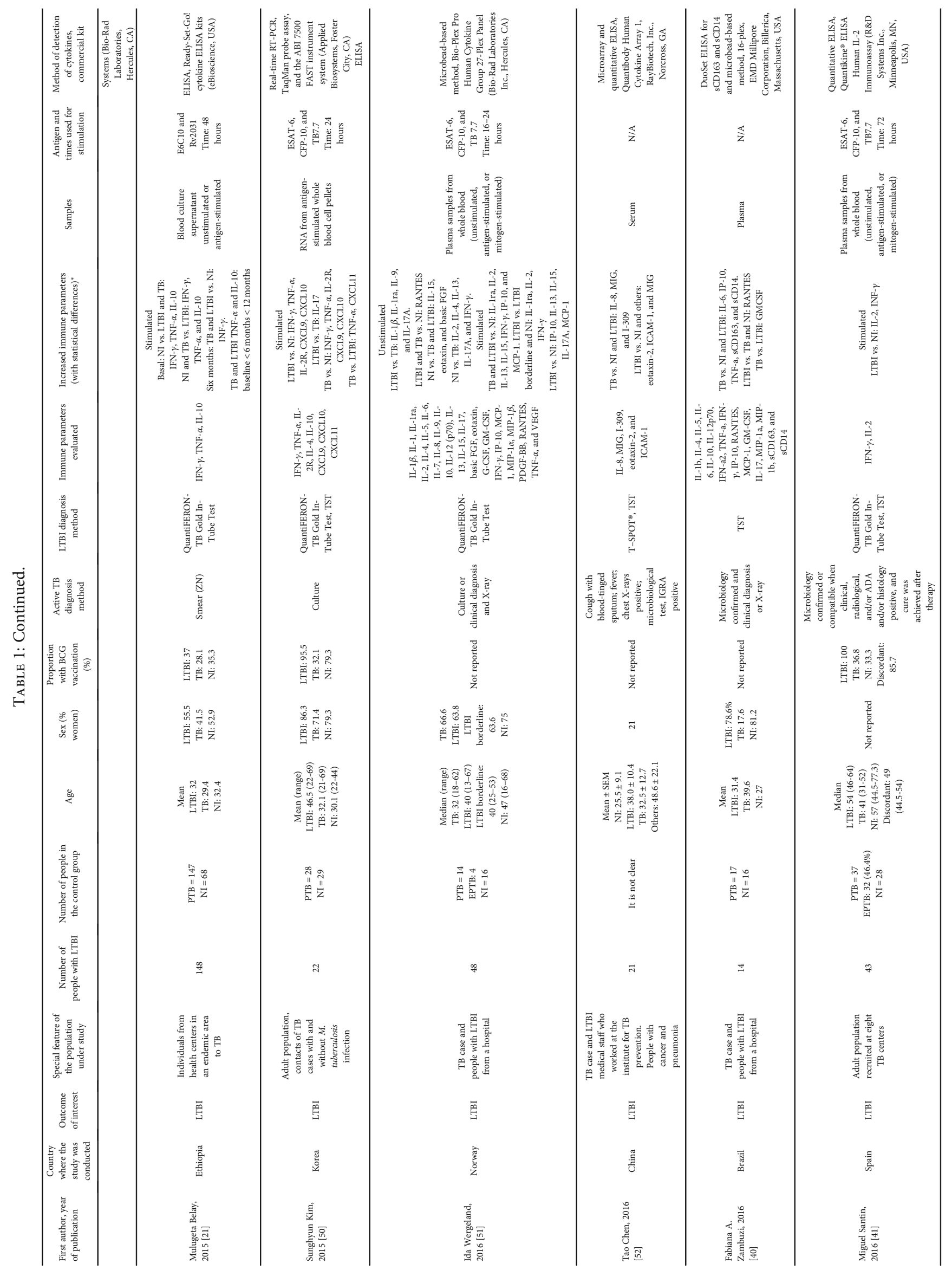




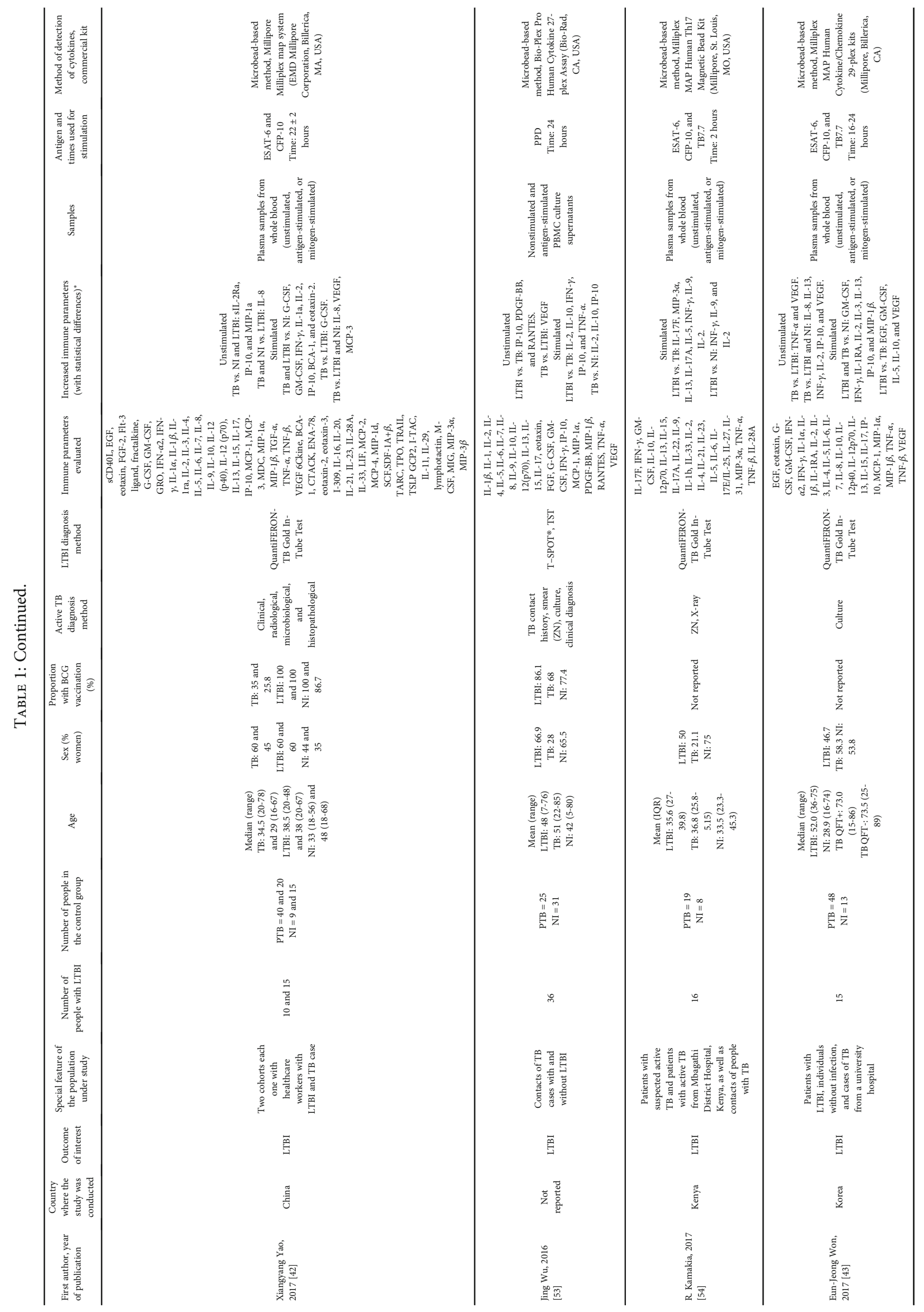







TABLE 2: Substances evaluated for ability to differentiate people with active and latent tuberculosis and uninfected with TB.

\begin{tabular}{|c|c|}
\hline Interleukins & $\begin{array}{l}\text { IL-1, IL-1 } \alpha \text {, IL-1 } \beta \text {, IL-2, IL-3, IL-4, IL-5, IL-6, IL-7, IL-8, IL-9, IL-10, IL-12, IL-12 (p40/70), IL-12 (p40), IL-12 } \\
\text { (p70), IL-13, IL-15, IL-17, IL-17A, IL-17E, IL-17F, IL-18, IL-21, IL-22, IL-23, IL-25, IL-27, IL-28A, IL-31, IL-33 }\end{array}$ \\
\hline Growth factors & $\begin{array}{c}\text { PDGF, PDGF-BB, EGF, PGF2 } \alpha \text {, FGF, TGF- } \alpha \text {, TGF- } \beta 1 \text {, G-CSF, CSF2, CSF3, GM-CSF, VEGF, VEGF-A, SFC, } \\
\beta \text {-NGF, basic FGF }\end{array}$ \\
\hline Interferon & INF- $\gamma$, IFN- $\alpha$, IFN- $\alpha 2$ \\
\hline Receptors & IL-1RA, IL-2R, sIL-2R $\alpha$, TNF- $\alpha$ R, IL-1R1, IL-1R2 \\
\hline Tumor necrosis factors & TNF- $\alpha$, TNF- $\beta$, TNF-SF10 (TRAIL) \\
\hline Alpha chemokines & $\begin{array}{l}\text { CXCL5 (ENA-78), CXCL6 (GCP-2/LIX), CXCL8 (IL-8), CXCL9 (MIG), CXCL10 (IP-10), CXCL11 (I-TAC), } \\
\text { CXCL12 (SDF-1 } \alpha+\beta) \text {, CXCL13 (BCA-1) }\end{array}$ \\
\hline Beta chemokines & $\begin{array}{l}\text { CCL1 (I-309), CCL2 (MCP-1), CCL3 (MIP-1 } \alpha) \text {, CCL4 (MIP-1 } \beta), \text { CCL5 (RANTES), CCL7 (MCP-3), } \\
\text { CCL8 (MCP-2), CCL11 (eotaxin), CCL13 (MCP-4), CCL15 (MIP-1 } \delta), \text { CCL17 (TARC), CCL20 (MIP-3 } \alpha \text { ), } \\
\text { CCL21 (6Ckine), CCL24 (eotaxin-2), CCL26 (eotaxin-3), CCL27 (CTACK) }\end{array}$ \\
\hline Delta chemokines & CX3CL1 (fractalkine) \\
\hline Others & ICAM-1 (CD54), sCD163, sCD14, sCD40L, I-TAG, MIF, LIF, LXA4, 15-Epi-LXA4 \\
\hline
\end{tabular}

The stimulation antigens used were ESAT-6, PPD, CFP10, TB7.7 (Rv2654), TB10.4, PE35 (Rv3872), PPE68 (Rv3873), Rv262, FbpB, E6C10, Rv2031, protein fraction 11_24 (Rv2626c), H37Rv soluble antigens, DosR Rv1737c, Rv2029c, Rv2628, Rpf Rv0867c Rv2389c, and inactivated bacteria (Table 1).

The concentration of biomarkers involved in the immune response is dependent on the type of protocol used for in vitro stimulation and the sample evaluated and has high variability between studies. Among the five substances exclusively elevated in LTBI and the two elevated in uninfected individuals, there was inconsistency in the samples processed throughout the studies. For example, IL-5 was evaluated in plasma (1 article), plasma samples from stimulated whole blood (11 articles), PBMC culture supernatant (3 articles), and blood culture supernatant ( 1 article) but was only increased in two papers that used plasma samples from stimulated whole blood (2/11 articles). In the case of active TB, one substance (CCL1/I-309) was elevated in four different sample types, one (IL-7) in three sample types, and the rest in two (usually plasma samples from whole blood unstimulated and stimulated) or one sample type.

ELISA $(n=17)$ and microbead-based method $(n=20)$ were the most frequently used methods for IP measurement (Table 1). Some of the articles used both methods. Table 1 describes details regarding laboratory measurements.

3.7. Risk of Bias of Included Articles. Of the 36 articles reviewed using the Newcastle-Ottawa scales and the Quality Assessment Scale for NIH observational studies, 7 articles had low risk of bias, 29 moderate, and none high risk of bias. Only one study out of the 36 performed calculation of the sample size and took into account the statistical power of their results (Additional files 3 and 4).

3.8. Biases. The main bias identified in the articles reviewed was the absence of a second administration of the tuberculin skin test to detect a possible booster effect, thus leading to the potential inclusion of individuals with false negative results of the TST. Another bias was the analysis of patients with pulmonary and extrapulmonary $\mathrm{TB}$ in the same group of active $\mathrm{TB}$ as the underlying immune competence and immune response may vary between localized or disseminated disease. In addition, children and adults were included in some studies; however, the analysis was not stratified for each population. Finally, patients with pulmonary TB were included in different phases of treatment; some studies included individuals that completed treatment at the time of IP measurement. The declining microbial burden during or at the end of therapy may contribute to false negative results (Additional files 3 and 4).

Only 4 of the reports took into account the study origin and population's ethnicity as a confounding factor, and these were evaluated by self-reported ethnicity [28, 38, 48, 52].

Of the included manuscripts, 27 articles provided a declaration of conflicts of interest (S3 and S4).

\section{Discussion}

The immune response against infection and disease caused by $M$. tuberculosis is mainly mediated by the recruitment and activation of $\mathrm{T}$ cells and macrophages, which in turn are regulated by multiple immune mediators such as interleukins and chemokines, possessing a diverse pro- and antiinflammatory property. The success of the immune response in halting the acquisition of $M$. tuberculosis is influenced by a myriad of environmental, microbial, and host factors. The host response is measured in order to determine $M$. tuberculosis infection in the form of skin tests or IGRAs, but this approach is limited by the inability to differentiate LTBI from active TB infection. The ability to refine diagnostics by using assays that incorporate measurement of multiple biomarkers will be critical in order to stride towards TB control and eventual elimination.

Most of the studies analyzed in this review focused on the main pro- and anti-inflammatory interleukins involved in the immune response, mediated mainly by Th1 and Th2 lymphocytes; a few others expanded the markers measured to include chemokine-like substances, growth factors, and receptors as part of the search for new diagnostic biomarkers that can discriminate between LTBI and active TB. 


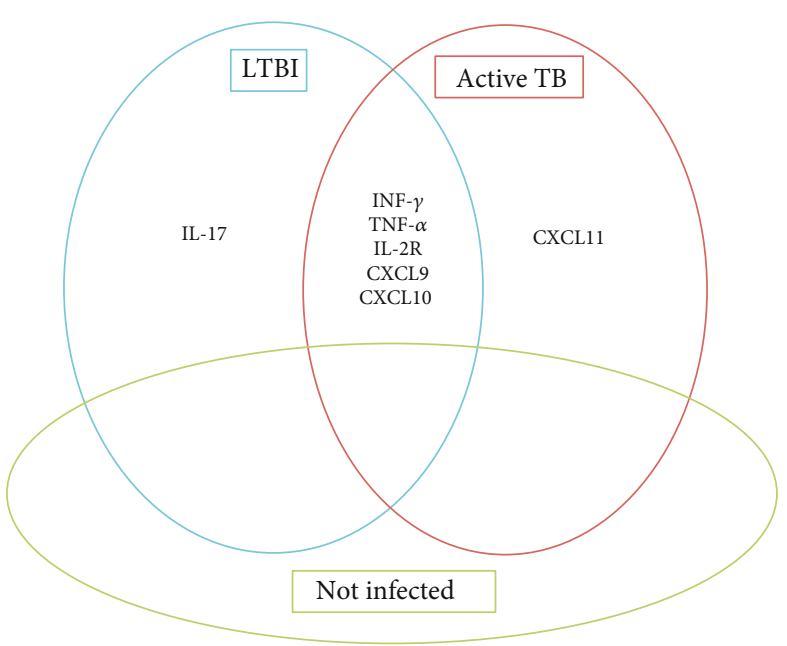

(a) RNA from antigen stimulated from whole blood cell pellets

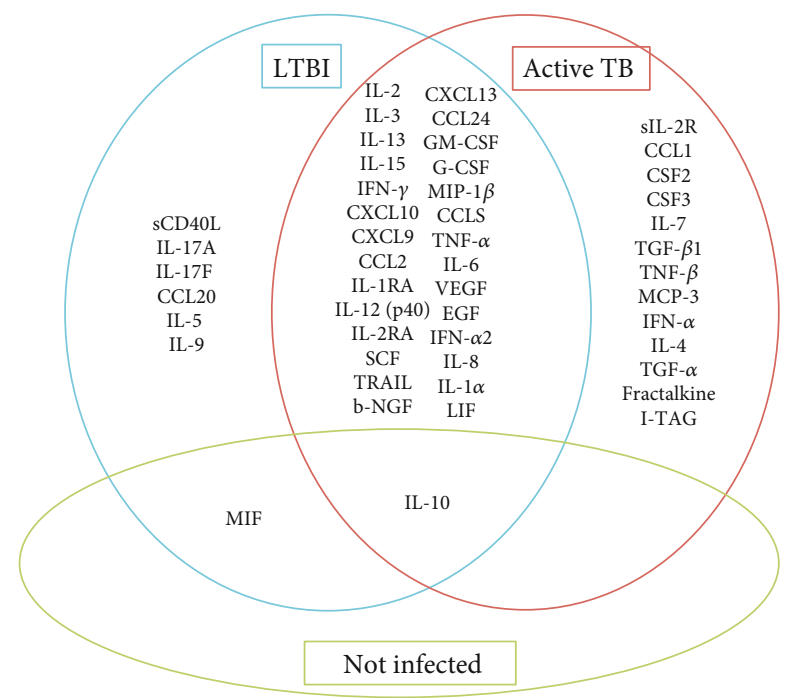

(c) Plasma samples from whole blood-stimulated

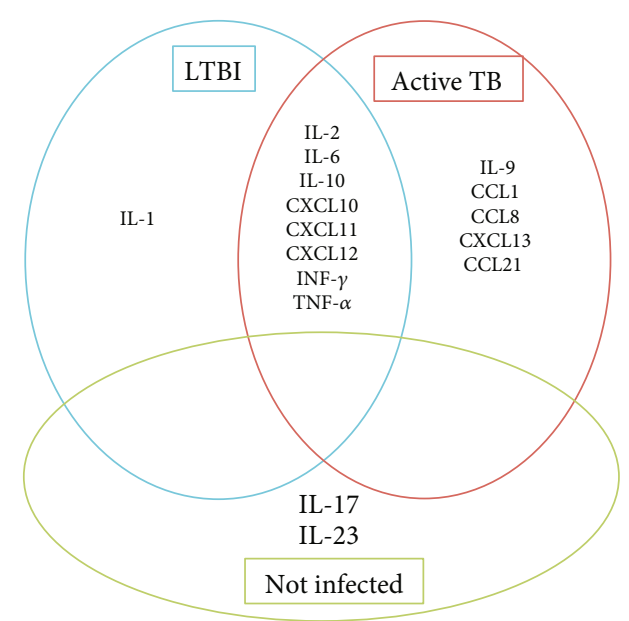

(e) PBMC culture supernatant-stimulated

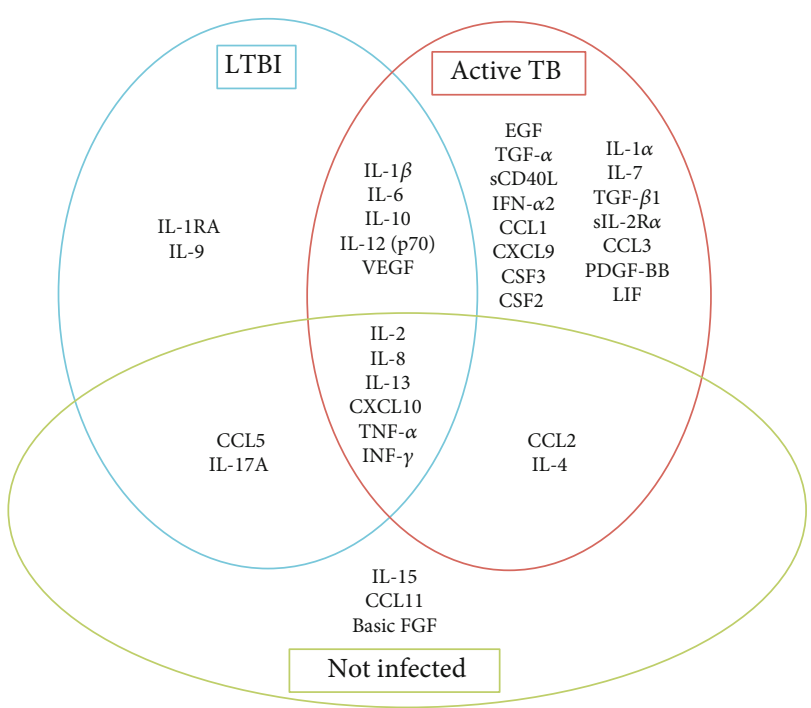

(b) Plasma samples from whole blood-unstimulated

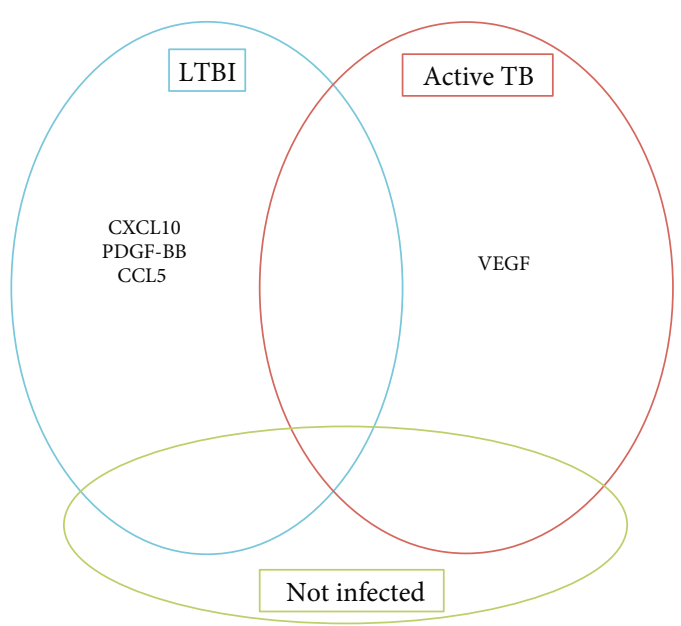

(d) PBMC culture supernatant-unstimulated

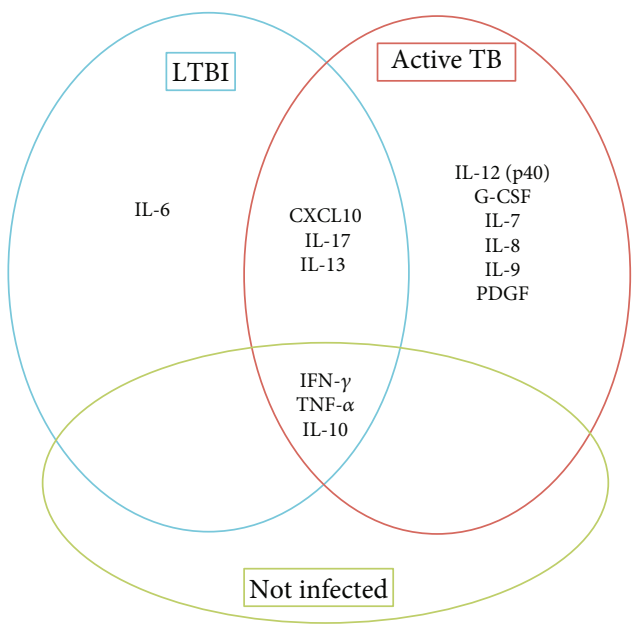

(f) Blood culture supernatant

Figure 2: Continued. 


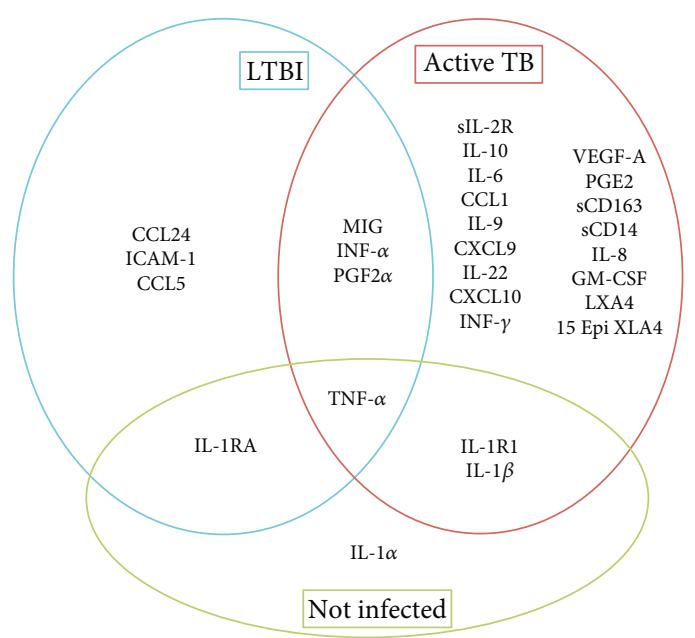

(g) Serum/plasma

Figure 2: (a-g) Immune cytokine/chemokine mediators statistically different reported in active TB, latent tuberculosis infection, and noninfected individuals in each sample type.

Several immune mediators in addition to INF- $\gamma$ have been identified. The most frequently evaluated markers are the cytokines IL-6, IL-10, IL-2, TNF- $\alpha$, and IP-10. Although the response to TB is reliant on Th1 (TNF- $\alpha$, INF- $\gamma$, and IL-2), this has been expanded by the addition of Th2 signature cytokine profile such as IL-6 and IL-10.

Elevated immune mediators and markers that were only detected in active TB share chemoattractant functions involved in trafficking of cells involved in the immune response, among which are $\mathrm{T}$ lymphocytes (CD4+ and CD8 $+)$, macrophages, dendritic cells, basophils, and eosinophils. These cytokines affect cell growth, maturation, and differentiation (Additional file 5). In LTBI, only interleukins IL17F and IL-5, associated with effector T cell profiles, are overexpressed. The effect of the cytokines found overexpressed in LTBI is related to the increased production of immune substances, chemoattraction, multiplication, and activation of lymphoid cells [56]. Of the cytokines identified in the systematic review, three (IL-12 and TGF- $\beta$ for active TB and IL-23 for uninfected individuals) are well-established markers involved in immune response to mycobacterial infection (Figure 3: available at http://www.genome.jp/kegg/pathway .html) [57].

While most attention has been directed to immune cells, some of the immune substances that participate in the response to $M$. tuberculosis are produced by epithelial cells, which play a fundamental role in the initiation and expansion of host defense mechanisms in the lung, providing protection against mycobacteria. Epithelial cells participate in activation of innate immunity, as well as adaptive immunity, inducing the recruitment and activation of dendritic cells and $\mathrm{T}$ and $\mathrm{B}$ lymphocytes, which in turn increase antigen recognition and production of antibodies and other immune substances $[58,59]$. These markers merit further investigation for the ability to distinguish early and late mycobacterial infection.

Despite some signals suggesting that the biomarker expression differences between LTBI and active TB can be used for diagnostics, choosing a panel of reproducible, discriminatory markers based on the results of the studies analyzed is quite difficult due to

(1) failure to account for the time/illness of the individuals studied. Not surprisingly, the biology of TB is much more complex than previously thought, and therefore, classification in LTBI and active TB is insufficient. What is considered LTBI actually corresponds to a range of infection status, which may have been recently acquired or present for decades. Recently acquired TB is associated with a higher progression rate to active disease pointing to distinct biological properties. The study of pulmonary immune substances in the animal model reveals changes in the expression of cytokines/chemokines in the cells that make up the granuloma. The diversity of granulomas (diverse functions and architectures and microenvironments) has consequences on the bacterial control $[60,61]$. It is suggested that after the in vitro stimulation, changes in the cellular expression due to the phase of infection or tuberculosis disease can lead to a varied response that is evidenced in the analyzed studies. Among the papers included, there were 22 different antigens used for in vitro stimulation, with concentrations that widely varied within the same immune factors and within the same group of patients; for example, INF- $\gamma$ ranges from 0 to $2640 \mathrm{pg} / \mathrm{ml}$, with overlapping concentrations between the uninfected individuals, LTBI, and active $\mathrm{TB}$ group, independent of the sample used (Additional file 6).

Given the heterogeneity of the biology of disease associated with LTBI, the ability to identify the duration of infection remains a challenge for future research. Inability to determine the duration and type of LTBI (i.e., what type of granuloma) might modify the observed response to a mycobacterial antigen 


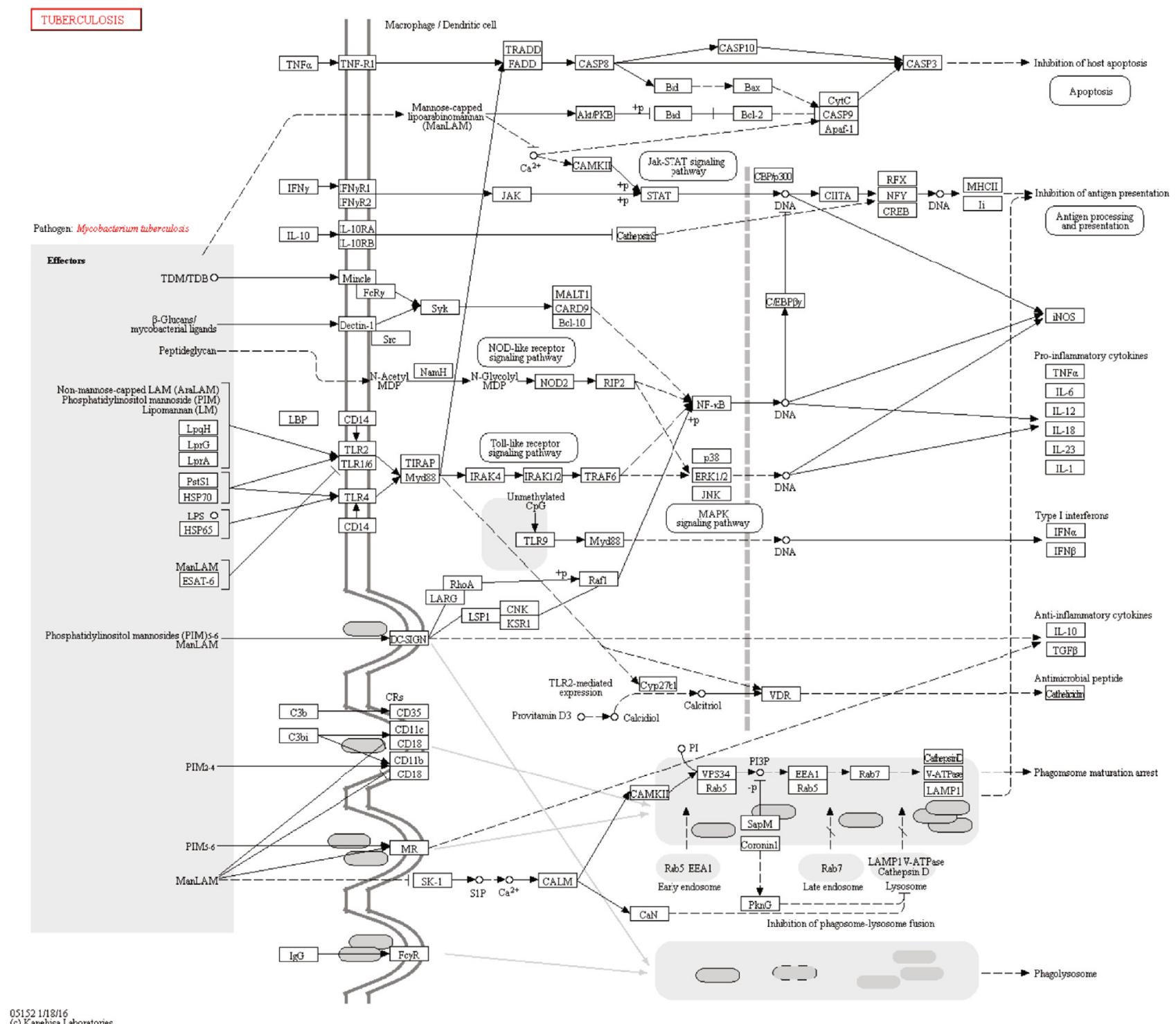

FIGURE 3: KEGG pathway, highlighting some of the pathways and mediators identified in the reviewed studies. The use of this figure was granted by copyright permission of KEGG [57], and the journal has a copy of the approval.

leading to blurring of the ability to interpret differences between study groups $[60,61]$.

(2) different samples and varied cell stimulation protocols. The samples used for the studies were predominantly plasma; however, culture supernatant, serum, and RNA were used introducing variability in measured concentration caused by the matrix used (Table 1 and Additional file 6). Several potential reasons for the variation in immune substance concentrations in plasma and serum from whole blood include inhibition of detection for specific cytokines (e.g., EGF, GM-CSF, IL-3, and IL-4) in the serum [62]; delay in processing of serum or plasma, sample hemolysis, presence of debris, or freeze-thaw cycles, all of which can adversely affect cytokine detection [63]; and the release of several mediators by platelets which can increase cytokine serum levels, especially CCL5 and CD40L [64].

In addition, the wide variety of antigens (ESAT-6, CFP-10, TB7.7, PPD, or Mtb CFA, among others) used to stimulate cells and different incubation times leads to the increases or decreases of the time of cellular exposure to the stimulus and therefore the concentration of the detected immune mediators and other substances. In addition, cellular stimulation adds complexity to the diagnostic utility of detecting biomarkers, especially in areas with limited laboratory infrastructure or access, as is the situation in many of the countries or settings where $\mathrm{TB}$ is endemic. In addition, reviewed articles show variations in the results due to the antigen used for stimulation [11-13]. In experiments with whole bacteria, it 
has been demonstrated that the strain used to carry out stimulation modifies the type of immune response in vitro; for example, the most recent strains in the M. tuberculosis lineage show a lower inflammatory response in macrophages when compared to the older strains [65]. Likewise, Leyten et al. evaluated 25 antigens of latency related to the DosR regulator of M. tuberculosis; it was observed that different antigens can give different cellular responses (measured by the production of INF- $\gamma$ ) after in vitro stimulation, and in addition, this can vary between healthy people, LTBI and active TB cases [11]. This limitation can be overcome in longitudinal studies applying the same measurement at different times along the natural history of $M$. tuberculosis infection.

(3) the variety of laboratory methods used for detection of substances, which in turn leads to the variable units of measurement and assay sensitivity. The ability to compare the heterogeneous samples is further compounded by use of ELISA, microbead assays, EIA, and real-time PCR - in the absence of an endogenous standard-yielding variable dynamic ranges [66]. The intraindividual variability cannot be assessed, as only 2 studies were longitudinal. This variability results in difficulty to compare and quantify studies

(4) the presence of a selection bias for nonapplication of the booster when individuals are screened using the tuberculin skin test. It is known that the booster effect can occur in individuals and is only detected when a second TST is applied to negative individuals between 1 and 4 weeks after the first administration. The increase in the frequency of positive individuals is notable in the population without any underlying diseases (in prisoners, an increase in positivity was reported from $66 \%$ to $77.6 \%$ [67]) or in those with other disorders such as rheumatoid arthritis (where the booster positivity changed from $31.3 \%$ and $21.7 \%$ to $46.5 \%$ and $28.8 \%$ in early and late rheumatoid arthritis, respectively) [68]. The lack of application of two-step TST may lead to erroneous classification to the uninfected group, resulting in false negative results [67-70]. Equally important, LTBI diagnoses were done using TST and/or IGRAs, which can introduce heterogeneity within the results. Indeed, in many studies when both methods were used, the results demonstrated inconsistent findings, a common theme discussed in literature [71-73]. Additionally, although some articles used TST for LTBI diagnosis, they did not consider the rate of BCG vaccination within children under 10 years old in their analyses [74]

(5) the fact that none of the studies adjusted the analysis for the effect of ethnicity on the association between IP concentrations and the different stages of TB. A study published by Coussens et al. reported that the inflammatory profile differs according to ancestry. Individuals of African descent with $\mathrm{TB}$, despite having similar mycobacterial strains and similar sociodemographic and clinical characteristics, have a different inflammatory profile compared to Eurasian patients with the same disease [75]. Similarly, Mwantembe et al. reported ethnic variation of cytokines (IL-1RA, IL-12) and chemokines (CCL2, CCL5, CCL11, and CXCL8) in South African patients with inflammatory bowel disease [76]. The concentrations of these chemokines and cytokines are determined by allelic frequency and have been involved in response to $M$. tuberculosis infection. Likewise, genes coding for proteins such as CCL2 [77], IL-17F, IL-17A [78], and IL-12 [37, 79] have been described as polymorphic; variation in allele frequency is affected by ethnic variation, affecting the antimycobacterial response, and thus may be driving the higher risk for development of TB among different populations. The examples emphasize the importance to adjust by ethnicity of the population at the time of reporting the results as these clearly impact biomarker concentrations.

Ethnicity is also related to the response to current M. tuberculosis infection screening tests. Genetic variants associated with the reaction to TST and IGRAs have been described. The TST1 locus is associated with a TST positivity per se (TST1 on 11p14), and the TST2 locus is associated with the intensity of TST reactivity (TST2 on 5p15) [3]. On the other hand, the production of INF- $\gamma$ has been associated with genetic factors such as the locus located in chromosomal regions $8 \mathrm{q} 12-22 \mathrm{n}$ and 3q13-22 [2]. The ethnicity must be considered when performing the immunological analysis in further research.

(6) the heterogeneity of the populations studied. First, the comparison group of active TB included patients with pulmonary TB and extrapulmonary TB together. In these two groups of patients, the presentation of the disease is different, and the main factors of innate immunity, cytokines and chemokines, which play a role in cell-mediated immunity, involved in the dissemination of M. tuberculosis, differ. Mutations have been reported in genes encoding the INF- $\gamma$ receptor, the IL-12 receptor, and the transcription-activating signal1 (STAT-1) in patients with extrapulmonary TB. Likewise, Yang et al. reported that there are differences in the immunopathogenicity of pulmonary and extrapulmonary infections. The production of CCL2, CXCL9, and CXCL8 modifies the type of tuberculous disease that a patient has, and they play a special role in the formation of granuloma [80]. Patients with pulmonary TB showed lower levels of the cytokines studied than those with extrapulmonary TB. CXCL8 concentration was found to be elevated in fatal TB, increases in CCL2 were observed with disseminated and meningeal TB [81], and TGF- $\beta$ increased in extrapulmonary TB in children compared to pulmonary TB [14]. 
Secondly, patients with active TB included in the studies were at different phases of treatment (before, during, and after completion of antituberculous therapy). Several studies have been carried out with the aim of evaluating new biomarkers that allow monitoring the patient's condition after initiating antituberculous therapy. Several of the studies were longitudinal, making it evident that the immune substances changed during the administration of $\mathrm{TB}$ treatment $[82,83]$. Changes in lung bacterial load related to treatment administration would appear to influence the concentration of cytokines detected in nonstimulated cells, with 17 out of the 27 cytokines/chemokines analyzed (IL- $1 \beta$, IL-2, IL-4, IL-5, IL-6, IL-9, IL-13, IL-17, eotaxin, IFN- $\gamma$, IP-10, MCP-1, MIP- $1 \alpha$, MIP-1 $\beta$, PDGF, RANTES, and VEGF) being significantly lower in patients with higher bacterial load and levels of IL10, IL15, and TNF- $\alpha$ being higher in the same patients [84].

\section{Conclusions}

Identification of biomarkers that individually or in combination can differentiate LTBI and active TB has been a research priority; however, a constellation of markers that differentiate between infection and disease is not yet available. The advances in high-throughput technologies for biomarker measurement are promising, but the variability of studies and potential biases that we have highlighted undermines the ability to identify reproducible markers. Although five parameters were exclusively increased in LTBI and 24 in active TB, only a single substance was consistently differential. These substances were not measured in all studies, and results are inconsistent between study groups, prohibiting the desired classification. Undoubtedly, the study of multiple immune substances seems to give better results than the study of a single biomarker; consequently, the search for immune profiles with multiple immune substances should be the goal of future research.

For the results obtained with different immune markers, future research should "harmonize" the methodological conditions to evaluate immune markers as the first step to draw any conclusion about LTBI parameter(s) for use as a diagnostic test. Those aspects include the presence of the booster effect, clinical classification of TB, the ethnicity of participants, and sample size estimation. In addition, cohort studies will allow identification of immune substances related to progression to active TB and conversion to LTBI and variations in the immune response due to the individual's stage of TB, measurement variation for cytokine/chemokines, and hormonal influences $[85,86]$.

The BCG has been associated with modulating the host's immune system and granting protection against MTB infection and disease $[87,88]$. BCG vaccination could potentially modify the concentration of the immune substances in vaccinated adults, considering that it changes the concentrations in children and adolescents [89]. Further studies should evaluate the effect of BCG vaccination in the immune marker response.
It is important to note that our review did not include HIV-infected populations or any other types of immunosuppression, nor children, since those populations have several confounders and particular characteristics that need to be analyzed separately and are beyond the scope of the present review. In addition, as the main goal of our paper was to identify immune markers associated with LTBI, we did not include articles that consider biomarkers for TB before and after the treatment.

$\begin{array}{ll}\text { Abbreviations } \\ \text { TB: } & \text { Tuberculosis } \\ \text { LTBI: } & \text { Latent tuberculosis infection } \\ \text { EPTB: } & \text { Extrapulmonary TB } \\ \text { PTB: } & \text { Pulmonary TB } \\ \text { NI: } & \text { No } \\ \text { TST: } & \text { Tuberculin skin test } \\ \text { HIV: } & \text { Human immunodeficiency virus } \\ \text { NIH: } & \text { National Institutes of Health } \\ \text { IGRAs: } & \text { Interferon-gamma release assays } \\ \text { INF: } & \text { Interferon } \\ \text { IL: } & \text { Interleukins } \\ \text { TNF: } & \text { Tumor necrosis factor } \\ \text { PRISMA: } & \text { Preferred Reporting Items for Systematic reviews } \\ & \text { and Meta-Analyses } \\ \text { ESAT-6: } & \text { Early secretory antigenic target-6, Rv3875 } \\ \text { CFP-10: } & \text { Culture filtrate protein-10, Rv3874 } \\ \text { PPD: } & \text { Purified protein derivatives } \\ \text { IP: } & \text { Immune parameter. }\end{array}$

\section{Data Availability}

All data generated or analyzed during this study are included in this published article [and its Additional files 1, 2, 3, 45 and 6].

\section{Conflicts of Interest}

The authors declare that they have no competing of interests.

\section{Authors' Contributions}

$\mathrm{MH}, \mathrm{CV}$, and ZVR contributed substantially to the conception or design of the work. $\mathrm{MH}$ and $\mathrm{CV}$ acquired data. $\mathrm{MH}$, $\mathrm{CV}, \mathrm{YK}$, and ZVR analyzed and interpreted data; drafted the article and revised it critically for important intellectual content; had final approval of the version to be published; and agreed to be accountable for all aspects of the work in ensuring that questions related to the accuracy or integrity of any part of the work are appropriately investigated and resolved. Mariana Herrera and Cristian Vera contributed equally to this work.

\section{Acknowledgments}

This systematic review was funded by the Administrative Department of Science, Technology and Innovation (Colciencias), as part of the research project entitled "Host gene expression profile used to identify latent TB infection and 
the transition to active disease-Perfil de la expresión génica del hospedero para identificar tuberculosis latente y la transición a enfermedad activa," grant number: 121071249878 and the project "Pro-inflammatory patterns of cytokine/chemokine associated with latent tuberculosis in people deprived of liberty (PDL)," grant number: 639B-06/16-55 funded by Universidad Pontificia Bolivariana. Grupo de Epidemiología at Universidad de Antioquia paid the open access fee of this article.

\section{Supplementary Materials}

Additional file 1: search strategies conducted in the systematic review. Additional file 2: summary of excluded studies and the reasons. Additional file 3: quality of cohort studies (risk of bias evaluation). Additional file 4: quality of crosssectional studies (risk of bias evaluation). Additional file 5: immune factors, production cell, diana cell, and effect in different tuberculosis stages. Additional file 6: concentration of immune factors in persons with different tuberculosis stages in the papers included in the systematic review. (Supplementary Materials)

\section{References}

[1] M. Pai, C. M. Denkinger, S. V. Kik et al., "Gamma interferon release assays for detection of Mycobacterium tuberculosis infection," Clinical Microbiology Reviews, vol. 27, no. 1, pp. 3-20, 2014.

[2] F. Jabot-Hanin, A. Cobat, J. Feinberg et al., "Major loci on chromosomes $8 \mathrm{q}$ and $3 \mathrm{q}$ control interferon $\gamma$ production triggered by bacillus Calmette-Guerin and $6-\mathrm{kDa}$ early secretory antigen target, respectively, in various populations," The Journal of Infectious Diseases, vol. 213, no. 7, pp. 1173-1179, 2016.

[3] A. Cobat, C. J. Gallant, L. Simkin et al., "Two loci control tuberculin skin test reactivity in an area hyperendemic for tuberculosis," The Journal of Experimental Medicine, vol. 206, no. 12, pp. 2583-2591, 2009.

[4] J. L. Flynn, J. Chan, K. J. Triebold, D. K. Dalton, T. A. Stewart, and B. R. Bloom, "An essential role for interferon gamma in resistance to Mycobacterium tuberculosis infection," The Journal of Experimental Medicine, vol. 178, no. 6, pp. 2249-2254, 1993.

[5] WHO, TDR, "Priorities for tuberculosis research," October 2017, https://apps.who.int/iris/bitstream/10665/85888/1/978 9241505970_eng.pdf.

[6] M. A. Guillén, "Avances en el diagnóstico de la infección tuberculosa," Archivos de Bronconeumología, vol. 47, no. 10, pp. 521-530, 2011.

[7] Cellular and Molecular Immunology, 9thOctober 2017, https:// www.elsevier.com/books/cellular-and-molecular-immuno logy/abbas/978-0-323-47978-3.

[8] M. Suzukawa, S. Akashi, H. Nagai et al., "Combined analysis of IFN- $\gamma$, IL-2, IL-5, IL-10, IL-1RA and MCP-1 in QFT supernatant is useful for distinguishing active tuberculosis from latent infection," PLoS One, vol. 11, no. 4, article e0152483, 2016.

[9] K. M. Pollock, H. S. Whitworth, D. J. Montamat-Sicotte et al., "T-cell immunophenotyping distinguishes active from latent tuberculosis," The Journal of Infectious Diseases, vol. 208, no. 6, pp. 952-968, 2013.
[10] N. Arji, M. Busson, G. Iraqi et al., "The MCP-1 (CCL2) -2518 GG genotype is associated with protection against pulmonary tuberculosis in Moroccan patients," Journal of Infection in Developing Countries, vol. 6, no. 1, pp. 73-78, 2011.

[11] G. F. Black, B. A. Thiel, M. O. Ota et al., "Immunogenicity of novel DosR regulon-encoded candidate antigens of Mycobacterium tuberculosis in three high-burden populations in Africa," Clinical and Vaccine Immunology, vol. 16, no. 8, pp. 1203-1212, 2009.

[12] R. Hussain, A. Kaleem, F. Shahid et al., "Cytokine profiles using whole-blood assays can discriminate between tuberculosis patients and healthy endemic controls in a BCG-vaccinated population," Journal of Immunological Methods, vol. 264, no. 1-2, pp. 95-108, 2002.

[13] E. M. S. Leyten, M. Y. Lin, K. L. M. C. Franken et al., "Human $\mathrm{T}$-cell responses to 25 novel antigens encoded by genes of the dormancy regulon of Mycobacterium tuberculosis," Microbes and Infection, vol. 8, no. 8, pp. 2052-2060, 2006.

[14] N. Pavan Kumar, R. Anuradha, B. B. Andrade et al., "Circulating biomarkers of pulmonary and extrapulmonary tuberculosis in children," Clinical and Vaccine Immunology, vol. 20, no. 5, pp. 704-711, 2013.

[15] A. M. Abou-Setta, L. A. Beaupre, C. A. Jones et al., NewcastleOttawa Scale Assessment of Cohort Studies, Agency for Healthcare Research and Quality, US, 2011, October 2017, https:// www.ncbi.nlm.nih.gov/books/NBK56664/.

[16] "Quality assessment tool for observational cohort and crosssectional studies - NHLBI, NIH," October 2017, https://www. nhlbi.nih.gov/health-pro/guidelines/in-develop/cardiovas cular-risk-reduction/tools/cohort.

[17] A. R. Jadad, R. A. Moore, D. Carroll et al., "Assessing the quality of reports of randomized clinical trials: is blinding necessary?," Controlled Clinical Trials, vol. 17, no. 1, pp. 1-12, 1996.

[18] M. Pai, M. McCulloch, J. D. Gorman et al., "Systematic reviews and meta-analyses: an illustrated, step-by-step guide," National Medical Journal of India, vol. 17, no. 2, pp. 86-95, 2004.

[19] “9.5.1 what is heterogeneity?," November 2018, https:// handbook-5-1.cochrane.org/chapter_9/9_5_1_what_is_hete rogeneity.htm.

[20] D. Nonghanphithak, W. Reechaipichitkul, W. Namwat, V. Naranbhai, and K. Faksri, "Chemokines additional to IFN$\gamma$ can be used to differentiate among Mycobacterium tuberculosis infection possibilities and provide evidence of an early clearance phenotype," Tuberculosis, vol. 105, pp. 28-34, 2017.

[21] M. Belay, M. Legesse, A. Mihret et al., "Pro- and antiinflammatory cytokines against Rv2031 are elevated during latent tuberculosis: a study in cohorts of tuberculosis patients, household contacts and community controls in an endemic setting," PLoS One, vol. 10, no. 4, article e0124134, 2015.

[22] P. R. Bapat, A. A. Husain, H. F. Daginawala et al., "The assessment of cytokines in Quantiferon supernatants for the diagnosis of latent TB infection in a tribal population of Melghat, India," Journal of Infection and Public Health, vol. 8, no. 4, pp. 329-340, 2015.

[23] Z. T. Handzel, V. Barak, Y. Altman et al., "Increased Th1 and Th2 type cytokine production in patients with active tuberculosis," The Israel Medical Association Journal: IMAJ, vol. 9, no. 6, pp. 479-483, 2007.

[24] N. N. Chegou, G. F. Black, M. Kidd, P. D. van Helden, and G. Walzl, "Host markers in Quantiferon supernatants 
differentiate active TB from latent TB infection: preliminary report," BMC Pulmonary Medicine, vol. 9, no. 1, p. 21, 2009.

[25] R. Biselli, S. Mariotti, V. Sargentini et al., "Detection of interleukin-2 in addition to interferon- $\gamma$ discriminates active tuberculosis patients, latently infected individuals, and controls," Clinical Microbiology and Infection, vol. 16, no. 8, pp. 1282-1284, 2010.

[26] J. S. Sutherland, B. C. de Jong, D. J. Jeffries, I. M. Adetifa, and M. O. C. Ota, "Production of TNF- $\alpha$, IL-12 (p40) and IL-17 can discriminate between active TB disease and latent infection in a West African cohort," PLoS One, vol. 5, no. 8, article e12365, 2010.

[27] S. Babu, S. Q. Bhat, N. P. Kumar, V. Kumaraswami, and T. B. Nutman, "Regulatory T cells modulate Th17 responses in patients with positive tuberculin skin test results," The Journal of Infectious Diseases, vol. 201, no. 1, pp. 20-31, 2010.

[28] M. Frahm, N. D. Goswami, K. Owzar et al., "Discriminating between latent and active tuberculosis with multiple biomarker responses," Tuberculosis, vol. 91, no. 3, pp. 250-256, 2011.

[29] P.-A. Rubbo, N. Nagot, V. le Moing et al., "Multicytokine detection improves latent tuberculosis diagnosis in health care workers," Journal of Clinical Microbiology, vol. 50, no. 5, pp. 1711-1717, 2012

[30] S. Wang, N. Diao, C. Lu et al., "Evaluation of the diagnostic potential of IP-10 and IL-2 as biomarkers for the diagnosis of active and latent tuberculosis in a BCG-vaccinated population," PLoS One, vol. 7, no. 12, article e51338, 2012.

[31] Y. Yu, Y. Zhang, S. Hu et al., "Different patterns of cytokines and chemokines combined with IFN- $\gamma$ production reflect Mycobacterium tuberculosis infection and disease," PLoS One, vol. 7, no. 9, article e44944, 2012.

[32] N. N. Chegou, P. N. Essone, A. G. Loxton et al., "Potential of host markers produced by infection phase-dependent antigenstimulated cells for the diagnosis of tuberculosis in a highly endemic area," PLoS One, vol. 7, no. 6, article e38501, 2012.

[33] D. Anbarasu, C. P. Raja, and A. Raja, "Multiplex analysis of cytokines/chemokines as biomarkers that differentiate healthy contacts from tuberculosis patients in high endemic settings," Cytokine, vol. 61, no. 3, pp. 747-754, 2013.

[34] K. D. Mayer-Barber, B. B. Andrade, S. D. Oland et al., "Hostdirected therapy of tuberculosis based on interleukin-1 and type I interferon crosstalk," Nature, vol. 511, no. 7507, pp. 99-103, 2014.

[35] I. Sauzullo, C. M. Mastroianni, F. Mengoni et al., "Long-term IFN- $\gamma$ and IL-2 response for detection of latent tuberculosis infection in healthcare workers with discordant immunologic results," Journal of Immunological Methods, vol. 414, pp. 5157, 2014.

[36] K. Kim, R. Perera, D. B. A. Tan et al., "Circulating mycobacterial-reactive CD4+ T cells with an immunosuppressive phenotype are higher in active tuberculosis than latent tuberculosis infection," Tuberculosis, vol. 94, no. 5, pp. 494501,2014

[37] Y. H. Jeong, Y.-G. Hur, H. Lee et al., "Discrimination between active and latent tuberculosis based on ratio of antigen-specific to mitogen-induced IP-10 production," Journal of Clinical Microbiology, vol. 53, no. 2, pp. 504-510, 2015.

[38] M. Wei, Z. Y. Wu, J. H. Lin et al., "Regulation network of serum cytokines induced by tuberculosis-specific antigens reveals biomarkers for tuberculosis diagnosis," Genetics and Molecular Research, vol. 14, no. 4, pp. 17182-17192, 2015.

[39] J. Y. Lee, Y. W. Jung, I. Jeong et al., "Immune parameters differentiating active from latent tuberculosis infection in humans," Tuberculosis, vol. 95, no. 6, pp. 758-763, 2015.

[40] F. A. Zambuzi, P. M. Cardoso-Silva, M. S. Espindola et al., "Identification of promising plasma immune biomarkers to differentiate active pulmonary tuberculosis," Cytokine, vol. 88, pp. 99-107, 2016.

[41] M. Santin, F. Morandeira-Rego, F. Alcaide et al., "Detection of interleukin-2 is not useful for distinguishing between latent and active tuberculosis in clinical practice: a prospective cohort study," Clinical Microbiology and Infection, vol. 22, no. 12, pp. 1007.e1-1007.e5, 2016.

[42] X. Yao, Y. Liu, Y. Liu et al., "Multiplex analysis of plasma cytokines/chemokines showing different immune responses in active TB patients, latent TB infection and healthy participants," Tuberculosis, vol. 107, pp. 88-94, 2017.

[43] E.-J. Won, J.-H. Choi, Y.-N. Cho et al., "Biomarkers for discrimination between latent tuberculosis infection and active tuberculosis disease," The Journal of Infection, vol. 74, no. 3, pp. 281-293, 2017.

[44] L. Arroyo, D. Marín, K. L. M. C. Franken, T. H. M. Ottenhoff, and L. F. Barrera, "Potential of DosR and Rpf antigens from Mycobacterium tuberculosis to discriminate between latent and active tuberculosis in a tuberculosis endemic population of Medellin Colombia," BMC Infectious Diseases, vol. 18, no. 1, p. 26, 2018.

[45] S. Y. Kim, M. S. Park, Y. S. Kim et al., "The responses of multiple cytokines following incubation of whole blood from TB patients, latently infected individuals and controls with the TB antigens ESAT-6, CFP-10 and TB7.7," Scandinavian Journal of Immunology, vol. 76, no. 6, pp. 580-586, 2012.

[46] J. Y. Hong, G. S. Jung, H. Kim et al., "Efficacy of inducible protein 10 as a biomarker for the diagnosis of tuberculosis," International Journal of Infectious Diseases, vol. 16, no. 12, pp. e855-e859, 2012.

[47] Y.-G. Hur, P. Gorak-Stolinska, A. Ben-Smith et al., "Combination of cytokine responses indicative of latent TB and active TB in Malawian adults," PLoS One, vol. 8, no. 11, article e79742, 2013.

[48] B. Pourakbari, S. Mamishi, M. Marjani, M. Rasulinejad, S. Mariotti, and S. Mahmoudi, "Novel T-cell assays for the discrimination of active and latent tuberculosis infection: the diagnostic value of PPE family," Molecular Diagnosis \& Therapy, vol. 19, no. 5, pp. 309-316, 2015.

[49] Y.-G. Hur, Y. A. Kang, S.-H. Jang et al., "Adjunctive biomarkers for improving diagnosis of tuberculosis and monitoring therapeutic effects," The Journal of Infection, vol. 70, no. 4, pp. 346-355, 2015.

[50] S. Kim, H. Lee, H. Kim et al., "Diagnostic performance of a cytokine and IFN- $\gamma$-induced chemokine mRNA assay after Mycobacterium tuberculosis-specific antigen stimulation in whole blood from infected individuals," The Journal of molecular diagnostics : JMD, vol. 17, no. 1, pp. 90-99, 2015.

[51] I. Wergeland, J. Assmus, and A. M. Dyrhol-Riise, "Cytokine patterns in tuberculosis infection; IL-1ra, IL-2 and IP-10 differentiate borderline QuantiFERON-TB samples from uninfected controls," PLoS One, vol. 11, no. 9, article e0163848, 2016.

[52] T. Chen, Z. Li, L. Yu et al., "Profiling the human immune response to Mycobacterium tuberculosis by human cytokine 
array," Tuberculosis (Edinburgh, Scotland), vol. 97, pp. 108117, 2016.

[53] J. Wu, S. Wang, C. Lu et al., "Multiple cytokine responses in discriminating between active tuberculosis and latent tuberculosis infection," Tuberculosis, vol. 102, pp. 68-75, 2017.

[54] R. Kamakia, S. Kiazyk, J. Waruk et al., "Potential biomarkers associated with discrimination between latent and active pulmonary tuberculosis," The International Journal of Tuberculosis and Lung Disease, vol. 21, no. 3, pp. 278-285, 2017.

[55] M. P. La Manna, V. Orlando, P. L. Donni et al., "Identification of plasma biomarkers for discrimination between tuberculosis infection/disease and pulmonary non tuberculosis disease," PLoS One, vol. 13, no. 3, article e0192664, 2018.

[56] I. V. Lyadova and A. V. Panteleev, "Th1 and Th17 cells in tuberculosis: protection, pathology, and biomarkers," Mediators of Inflammation, vol. 2015, Article ID 854507, 13 pages, 2015.

[57] M. Kanehisa, M. Furumichi, M. Tanabe, Y. Sato, and K. Morishima, "KEGG: new perspectives on genomes, pathways, diseases and drugs," Nucleic Acids Research, vol. 45, no. D1, pp. D353-D361, 2017.

[58] Y. Li, Y. Wang, and X. Liu, "The role of airway epithelial cells in response to mycobacteria infection," Journal of Immunology Research, vol. 2012, Article ID 791392, 11 pages, 2012.

[59] N. E. Mvubu, B. Pillay, L. R. McKinnon, and M. Pillay, "Mycobacterium tuberculosis strains induce strain-specific cytokine and chemokine response in pulmonary epithelial cells," Cytokine, vol. 104, pp. 53-64, 2018.

[60] A. M. Cadena, S. M. Fortune, and J. A. L. Flynn, "Heterogeneity in tuberculosis," Nature Reviews Immunology, vol. 17, no. 11, pp. 691-702, 2017.

[61] C. E. Barry 3rd, H. I. Boshoff, V. Dartois et al., "The spectrum of latent tuberculosis: rethinking the biology and intervention strategies," Nature Reviews Microbiology, vol. 7, no. 12, pp. 845-855, 2009.

[62] Y. Rosenberg-Hasson, L. Hansmann, M. Liedtke, I. Herschmann, and H. T. Maecker, "Effects of serum and plasma matrices on multiplex immunoassays," Immunologic Research, vol. 58, no. 2-3, pp. 224-233, 2014.

[63] W. de Jager, K. Bourcier, G. T. Rijkers, B. J. Prakken, and V. Seyfert-Margolis, "Prerequisites for cytokine measurements in clinical trials with multiplex immunoassays," BMC Immunology, vol. 10, no. 1, p. 52, 2009.

[64] T. H. A. Tvedt, K. P. Rye, H. Reikvam, A. K. Brenner, and $\varnothing$. Bruserud, "The importance of sample collection when using single cytokine levels and systemic cytokine profiles as biomarkers - a comparative study of serum versus plasma samples," Journal of Immunological Methods, vol. 418, pp. 19-28, 2015.

[65] D. Portevin, S. Gagneux, I. Comas, and D. Young, "Human macrophage responses to clinical isolates from the Mycobacterium tuberculosis complex discriminate between ancient and modern lineages," PLoS Pathogens, vol. 7, no. 3, article e1001307, 2011.

[66] A. Ledur, C. Fitting, B. David, C. Hamberger, and J. M. Cavaillon, "Variable estimates of cytokine levels produced by commercial ELISA kits: results using international cytokine standards," Journal of Immunological Methods, vol. 186, no. 2, pp. 171-179, 1995.

[67] Z. V. Rueda, L. Arroyave, D. Marin et al., "High prevalence and risk factors associated with latent tuberculous infection in two
Colombian prisons," The International Journal of Tuberculosis and Lung Disease, vol. 18, no. 10, pp. 1166-1171, 2014.

[68] E. Dogan, R. Erkoc, H. Sayarlioglu, and K. Uzun, “Tuberculin skin test results and the booster phenomenon in two-step tuberculin skin testing in hemodialysis patients," Renal Failure, vol. 27, no. 4, pp. 425-428, 2005.

[69] L. Pérez-Barbosa, J. A. Esquivel-Valerio, A. C. Arana-Guajardo, D. Vega-Morales, J. Riega-Torres, and M. A. Garza-Elizondo, "Increased detection of latent tuberculosis by tuberculin skin test and booster phenomenon in early rheumatoid arthritis patients," Rheumatology International, vol. 35, no. 9, pp. 1555-1559, 2015.

[70] E. G. Teixeira, A. Kritski, A. Ruffino-Netto et al., "Two-step tuberculin skin test and booster phenomenon prevalence among Brazilian medical students," The International Journal of Tuberculosis and Lung Disease, vol. 12, no. 12, pp. 14071413, 2008.

[71] S. E. Dorman, R. Belknap, E. A. Graviss et al., "Interferon- $\gamma$ release assays and tuberculin skin testing for diagnosis of latent tuberculosis infection in healthcare workers in the United States," American Journal of Respiratory and Critical Care Medicine, vol. 189, no. 1, pp. 77-87, 2014.

[72] K. Overton, R. Varma, and J. J. Post, "Comparison of interferon- $\gamma$ release assays and the tuberculin skin test for diagnosis of tuberculosis in human immunodeficiency virus: a systematic review," Tuberculosis Respiratory Disease, vol. 81, no. 1, pp. 59-72, 2018.

[73] P. Lu, X. Chen, L.-M. Zhu, and H.-T. Yang, "Interferongamma release assays for the diagnosis of tuberculosis: a systematic review and meta-analysis," Lung, vol. 194, no. 3, pp. 447-458, 2016.

[74] J. D. Mancuso, R. M. Mody, C. H. Olsen, L. H. Harrison, M. Santosham, and N. E. Aronson, "The long-term effect of bacille Calmette-Guérin vaccination on tuberculin skin testing: a 55-year follow-up study," Chest, vol. 152, no. 2, pp. 282-294, 2017.

[75] A. K. Coussens, R. J. Wilkinson, V. Nikolayevskyy et al., "Ethnic variation in inflammatory profile in tuberculosis," PLoS Pathogens, vol. 9, no. 7, article e1003468, 2013.

[76] O. Mwantembe, M. C. Gaillard, M. Barkhuizen et al., "Ethnic differences in allelic associations of the interleukin-1 gene cluster in South African patients with inflammatory bowel disease (IBD) and in control individuals," Immunogenetics, vol. 52, no. 3-4, pp. 249-254, 2001.

[77] W. X. Feng, P. O. Flores-Villanueva, I. Mokrousov et al., "CCL2-2518 (A/G) polymorphisms and tuberculosis susceptibility: a meta-analysis," International Journal of Tuberculosis and Lung Disease, vol. 16, no. 2, pp. 150-156, 2012.

[78] J. Zhao, C. Wen, and M. Li, "Association analysis of interleukin-17 gene polymorphisms with the risk susceptibility to tuberculosis," Lung, vol. 194, no. 3, pp. 459-467, 2016.

[79] G. Liu, G. Li, Y. Xu et al., "Association between IL12B polymorphisms and tuberculosis risk: a meta-analysis," Infection, Genetics and Evolution, vol. 21, pp. 401-407, 2014.

[80] D. Yang and Y. Kong, "The bacterial and host factors associated with extrapulmonary dissemination of Mycobacterium tuberculosis," Frontiers of Biology, vol. 10, no. 3, pp. 252-261, 2015.

[81] Z. Hasan, B. Jamil, J. Khan et al., "Relationship between circulating levels of IFN- $\gamma$, IL-10, CXCL9 and CCL2 in pulmonary and extrapulmonary tuberculosis is dependent on disease 
severity," Scandinavian Journal of Immunology, vol. 69, no. 3, pp. 259-267, 2009.

[82] V. Clifford, C. Zufferey, A. Street, J. Denholm, M. Tebruegge, and N. Curtis, "Cytokines for monitoring anti-tuberculous therapy: a systematic review," Tuberculosis, vol. 95, no. 3, pp. 217-228, 2015.

[83] S. Ashenafi, G. Aderaye, A. Bekele et al., "Progression of clinical tuberculosis is associated with a Th2 immune response signature in combination with elevated levels of SOCS3," Clinical Immunology, vol. 151, no. 2, pp. 84-99, 2014.

[84] R. Heslop, A. L. Bojang, S. Jarju et al., "Changes in host cytokine patterns of TB patients with different bacterial loads detected using $16 \mathrm{~S}$ rRNA analysis," PLoS One, vol. 11, no. 12, article e0168272, 2016.

[85] Y. Z. Foo, S. Nakagawa, G. Rhodes, and L. W. Simmons, "The effects of sex hormones on immune function: a meta-analysis," Biological Reviews, vol. 92, no. 1, pp. 551-571, 2017.

[86] S. L. Klein and K. L. Flanagan, "Sex differences in immune responses," Nature Reviews. Immunology, vol. 16, no. 10, pp. 626-638, 2016.

[87] "PPD-induced monocyte mitochondrial damage is associated with a protective effect to develop tuberculosis in BCG vaccinated individuals: a cohort study," 2020, https://journals.plos. org/plosone/article?id=10.1371/journal.pone.0171930.

[88] A. L. Katelaris, C. Jackson, J. Southern et al., "Effectiveness of BCG vaccination against Mycobacterium tuberculosis infection in adults: a cross-sectional analysis of a UK-based cohort," The Journal of Infectious Diseases, vol. 221, no. 1, pp. 146-155, 2020.

[89] H. M. Dockrell, S. G. Smith, and M. K. Lalor, "Variability between countries in cytokine responses to BCG vaccination: what impact might this have on protection?," Expert Review of Vaccines, vol. 11, no. 2, pp. 121-124, 2012. 